\title{
Ultrastructure of high altitude pulmonary oedema
}

\author{
DONALD HEATH, HOMEIRA MOOSAVI, \\ and PAUL SMITH \\ Department of Pathology, University of Liverpool
}

\begin{abstract}
Heath, D., Moosavi, H., and Smith, P. (1973). Thorax, 28, 694-700. Ultrastructure of high altitude pulmonary oedema. When rats are exposed for 12 hours to simulated high altitude corresponding to the summit of Mount Everest, they develop ultrastructural changes in the lungs. These consist of the formation and protrusion of multiple endothelial vesicles into the pulmonary capillaries. It seems likely that they are associated with the development of high altitude pulmonary oedema.
\end{abstract}

Acute and rapidly progressive pulmonary oedema sometimes occurs in persons who ascend to a high altitude. It may afflict the unacclimatized subject who is exposed to diminished barometric pressure too quickly while engaged in strenuous physical exercise and is thus a hazard for the mountain climber and skier (Fred, Schmidt, Bates, and Hecht, 1962). It is also likely to develop in young people who live at high altitude and then return to the mountains after a short period at lower levels (Marticorena et al., 1964). Although this condition is well known and its clinical features are simply those of severe acute pulmonary oedema, the mechanism of its production is controversial. In the following experiment we attempted to establish if there are any ultrastructural features in the lung characteristic of the condition and to see if they are consistent with the haemodynamic disturbances known to arise in association with high altitude pulmonary oedema. For our animal model we selected rats in which acute pulmonary oedema was induced by exposure to simulated high altitude in a hypobaric chamber.

\section{METHOD AND MATERIALS}

Six adult Wistar albino rats were studied. Their sex and body weight are shown in the Table. Four of the rats (T1 to $T 4$ ) were placed in a hypobaric chamber and the pressure within it was reduced over a period of one hour to 265 torr. Thus they were exposed to a simulated high altitude corresponding approximately to the summit of Mount Everest. After 12 hours the pressure was returned to normal sea-level pressure (760 torr). The rats were removed from the chamber and killed immediately by cervical

Requests for reprints: Professor D. Heath, Department of Pathology, University of Liverpool
T A B L E

SEX AND BODY WEIGHT

\begin{tabular}{ccc|c|c}
\hline \multicolumn{3}{c|}{$\begin{array}{c}\text { Control (C) or } \\
\text { Test (T) Rat }\end{array}$} & Sex & Body Wt \\
(g)
\end{tabular}

dislocation without the use of any anaesthetic vapour. Two control rats $(\mathrm{C} 1$ and $\mathrm{C} 2)$ were also studied.

The thorax was opened and a small piece of the superior lobe of the right lung was removed and cut into blocks, $1 \mathrm{~mm}^{3}$ in volume. These were immediately fixed in ice-cold buffered glutaraldehyde and subsequently post-fixed in osmium tetroxide. They were then stained with uranyl acetate and embedded in Araldite. Thin sections, $1 \mu \mathrm{m}$, were cut with an LKB Ultrotome III ultramicrotome, mounted on glass slides and stained with toluidine blue for the selection of suitable areas for electron microscopy. The blocks were then trimmed and ultrathin sections were cut, stained with lead citrate, and examined in an AEI EM 6B electron microscope.

\section{RESULTS}

CLINICAL After being exposed to simulated high altitude the rats became inactive and lost interest in food. A pronounced tachypnoea developed and persisted throughout the 12 hours of exposure. Rat T1 showed a peculiar combination of coughing and kicking.

ELECTRON MICROSCOPY The lungs of all the test rats exposed to simulated high altitude showed the same ultrastructural changes. There were multiple extrusions into the pulmonary capillaries 
(Figs. 1, 2, and 3). These took the form of vesicles which contained a granular material and we interpret these as blebs filled with oedema fluid. When seen in longitudinal section these vesicles had an elongated shape which accommodated itself to the confines of the capillary into which it projected (Fig. 1). The extrusions were large enough to occlude pulmonary capillaries into which they projected (Fig. 1). Fortuitous sections revealed that the vesicles arose by pedicles from localized widened areas of the fused basement membranes of the alveolar wall where there appeared to be an accumulation of fluid (Figs. 1 and 2). The oedema vesicles were covered by an exceedingly thin layer of cytoplasm which consisted of part of the overlying endothelial cell of the

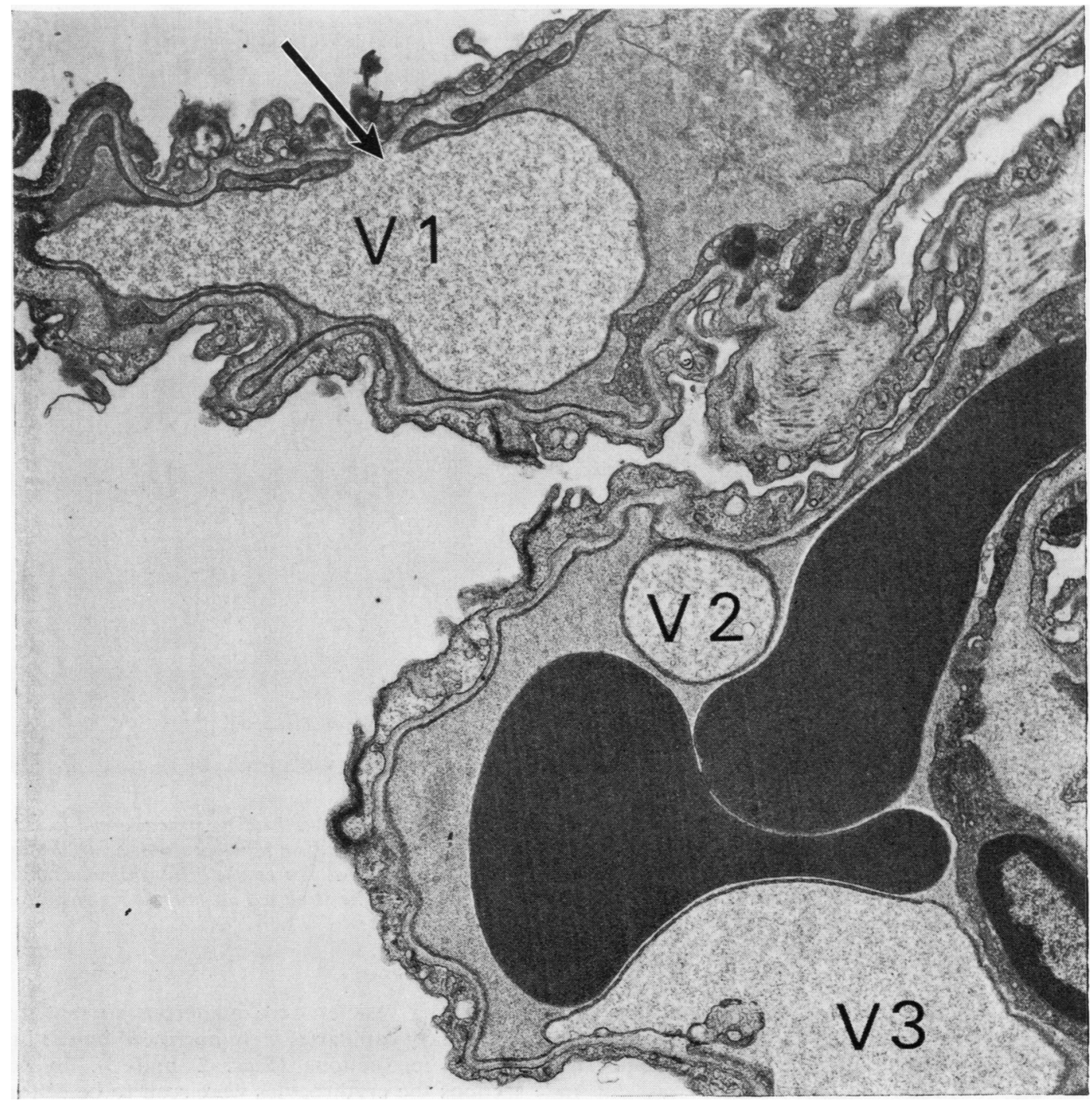

FIG. 1. Electron micrograph of two pulmonary capillaries from a test rat, the lower of which contains two erythrocytes appearing as large dark masses. Three endothelial vesicles, $V 1, V 2$, and $V 3$, are seen. The upper has been cut in longitudinal section and assumes the shape of the capillary into which it projects. Its pedicle is indicated by an arrow. The middle vesicle, V2, has been cut in transverse section and gives the spurious appearance of lying free in the capillary $(\times 12,500)$. 


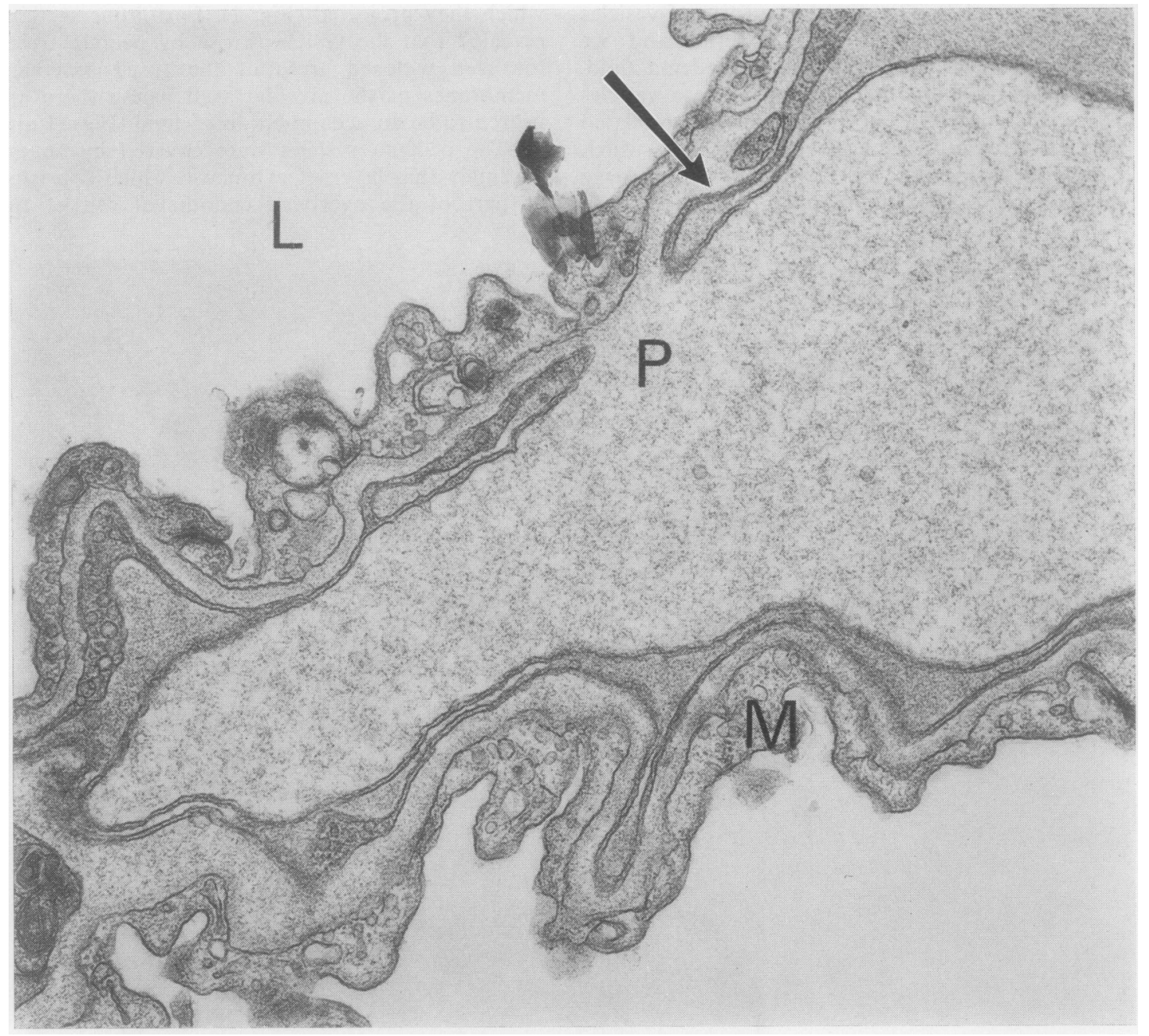

FIG. 2. The endothelial vesicle V1 from Fig. 1 at higher magnification to show details of its structure and formation. The fused basement membrane of the capillary wall is indicated by an arrow. Resting on it and projecting into the lumen, $L$, of the alveolus is the thin layer of cytoplasm of a membranous pneumocyte, $M$. The vesicle arises from the basement membrane by a pedicle, $P$. It is covered by a double membrane representing the stretched ultrathin layer of cytoplasm from an endothelial cell lining the capillary $(\times 25,000)$.

pulmonary capillary stretched by the localized accumulation of fluid (Fig. 2). This stretched layer of cytoplasm appeared as a double membrane (Fig. 2). When an oedema vesicle was cut in transverse section without fortuitously including its pedicle it appeared as a round body covered by a double membrane and gave the spurious appearance of lying free in the pulmonary capillary (Figs. 1 and 3).
Oedema vesicles were numerous so that many pulmonary capillaries were narrowed by the intraluminal projections (Figs. 1 and 3 ). In some sections the erythrocytes were seen to be distorted by the oedema vesicles which reduced the diameter of the capillary (Fig. 1). There was associated oedema coagulum within the alveolar spaces (Fig. 3).

The membranous type 1 pneumocytes lining the 


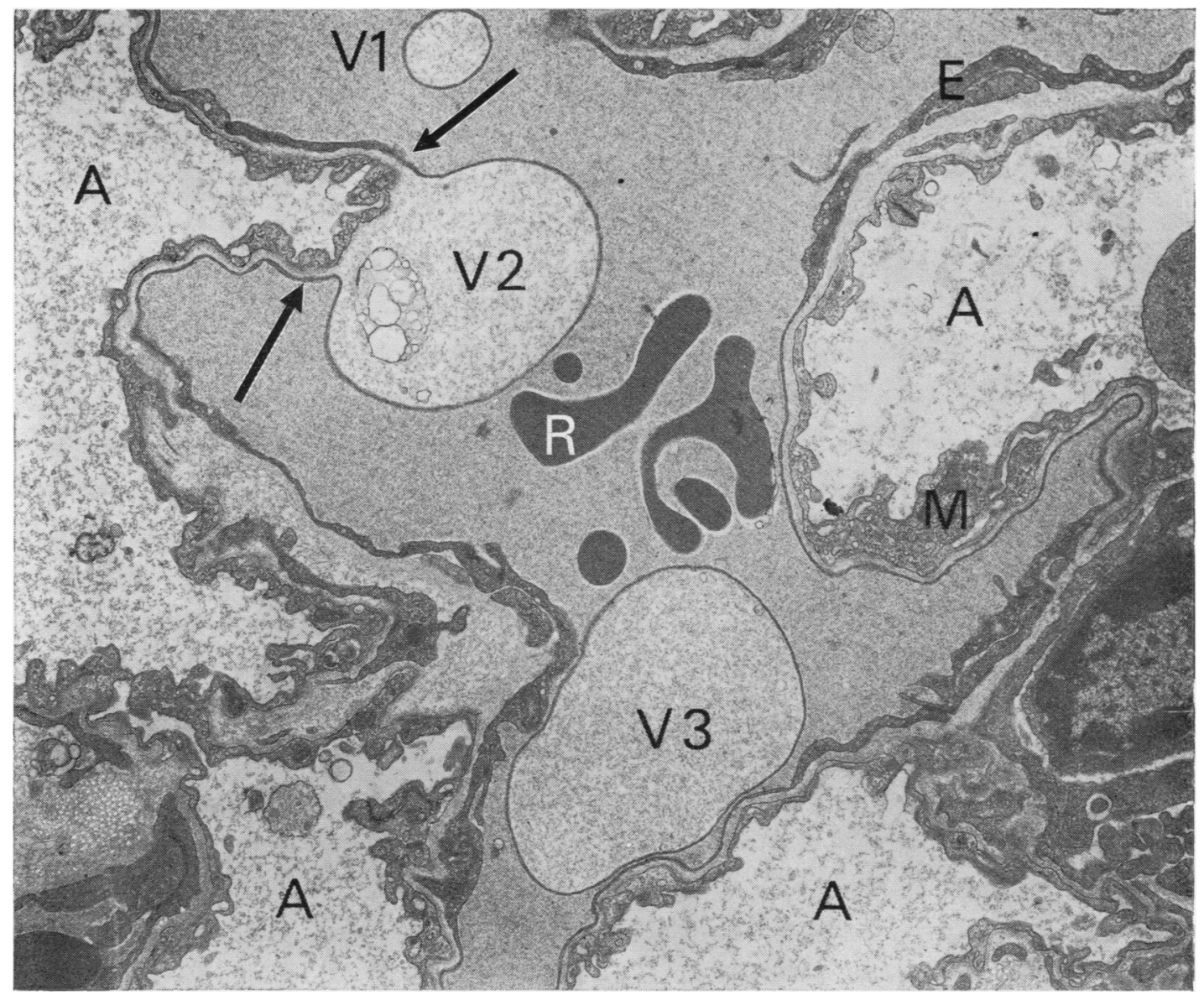

FIG. 3. Electron micrograph of pulmonary capillary from a test rat showing three oedematous vesicles, $V 1, V 2$, and $V 3$, projecting into its lumen. Vesicles $V 1$ and $V 3$ have been sectioned transversely and give the spurious impression of floating free in the plasma. The true nature and attachment of the vesicles are, however, shown in V2. In the area between the arrows the basement membrane has become grossly oedematous and expanded projecting as a vesicle into the capillary. The vesicle is covered by a thin granular layer which at the pedicle of the vesicle appears to be double-layered. This layer appears to be the stretched attenuated cytoplasm of endothelial cells of the pulmonary capillaries. The alveolar air spaces, A, contain oedema fluid appearing as granular material. $M=$ membranous pneumocyte; $R=$ red blood cell; $E=$ endothelial cell of pulmonary capillary $(\times 18,750)$.

alveolar spaces showed pronounced micropinocytosis with membrane-bound vesicles within their cytoplasm (Fig. 4). Their cytoplasm was swollen and pale showing the features of hydropic degeneration. The endothelial cells lining the pulmonary capillaries also contained many vesicles.

Pinocytosis ('cell drinking') is an appearance seen on light microscopy and seems to be pro- duced by a process by which the cell membrane can regulate the passage of substances into, and perhaps out of, the cell. A similar process, micropinocytosis, is thought to take place at ultrastructural level. It is indicated by small indentations or invaginations of the cell membrane, a few hundred Ångströms in diameter, often narrowed at their necks to form flask-shaped vesicles known as caveolae (Toner and Carr, 1971). 


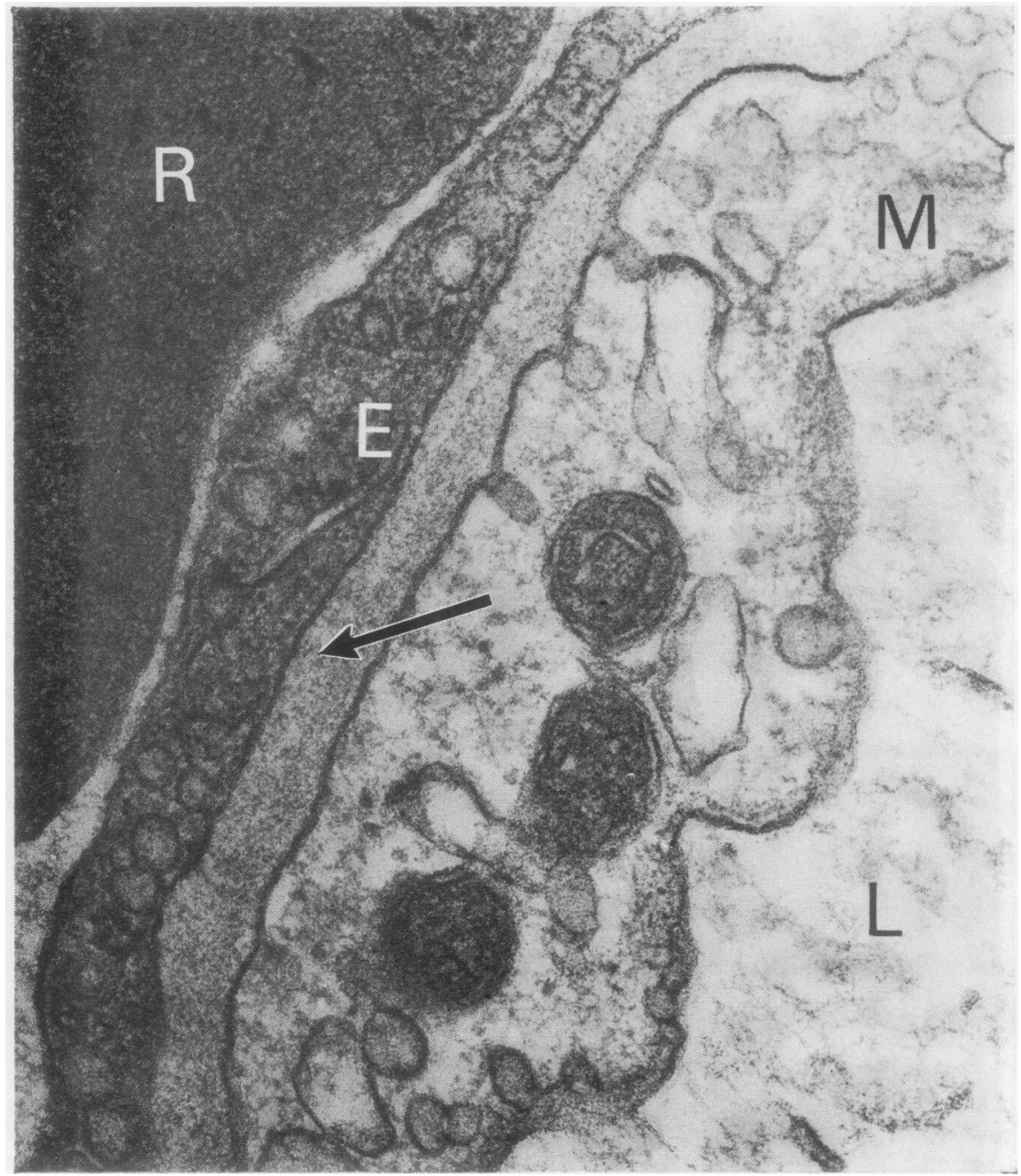

FIG. 4. Electron micrograph showing the components of the alveolar wall in one of the test rats. The lumen of the alveolar space, $L$, contains granular oedema coagulum. The fused basement membrane is indicated by an arrow. Its inner aspect is lined by an endothelial cell, $E$, and a red blood cell, $R$, lies adjacent in the lumen of the pulmonary capillary. The alveolar space is lined by a membranous pneumocyte, $M$. The membranous pneumocyte contains many caveolae, some of which open as cup-shaped structures onto the surface of the cell. Others appear to open as cup-shaped structures onto the basement membrane. It is swollen and pale and shows the features of hydropic vacuolation. The cytoplasm of the endothelial cell also contains many caveolae. $(\times 75,000)$.

\section{DISCUSSION}

People who develop high altitude pulmonary oedema are usually young and healthy, free of heart and lung disease, and are indeed often of athletic disposition. There is no suggestion of preceding respiratory infection. Some individuals and families appear to be particularly susceptible to the condition.

High altitude pulmonary oedema is likely to develop within 24 hours of the ascent and to follow prolonged effort. Initial symptoms of dry cough, dyspnoea, palpitations, precordial discomfort, headache, and nausea progress to severe 
respiratory distress. The subject becomes extremely breathless and coughs up foamy pink sputum. Weakness and vomiting ensue. Peripheral vasoconstriction follows with pallor of the face and coldness and clamminess of the skin. There may be cyanosis of the lips and nails. On examination there are widespread crepitations throughout the lungs. Administration of oxygen leads to rapid improvement but in the absence of such therapy the condition may be fatal. Radiologically there is evidence of pulmonary oedema with coarse mottling in both lung fields which is most prominent in the hilar regions and often spares the apices and bases of the lungs. The cardiac shadow remains of normal size and configuration throughout the episode. The electrocardiographic findings are those of acute right ventricular strain. Histological examination of the lung in a subject who has died of high altitude pulmonary oedema shows oedema coagulum in the alveolar spaces, hyaline membranes in contact with alveolar walls, and thickening of alveolar septa due to dilatation of pulmonary capillaries. Recent thrombi are sometimes seen in pulmonary arteries and septal capillaries (Marticorena et al., 1964 ; Fred et al., 1962).

Some insight into the mechanism of production of high altitude pulmonary oedema may be obtained from the results of cardiac catheterization carried out during an attack. Fred et al. (1962) studied the pulmonary haemodynamics of a 48-year-old doctor who had an attack of pulmonary oedema after two days of vigorous skiing at altitudes between 2,600 and $3,125 \mathrm{~m}$. They found a raised pulmonary arterial mean pressure of $46 \mathrm{mmHg}$ with normal left atrial and pulmonary venous pressure, the latter being obtained by advancing the catheter through a probe-patent foramen ovale into the left atrium and thence into a pulmonary vein. Pulmonary arterial hypertension at high altitude is usually associated with constriction of the terminal portions of the pulmonary arterial tree but as this constriction occurs proximal to the pulmonary capillary bed it would not explain the development of pronounced pulmonary oedema. Fred et al. argue that to explain the coexistence of acute pulmonary arterial hypertension and acute pulmonary oedema in the presence of normal left atrial and pulmonary venous pressure one must postulate some increased vascular resistance situated in either the pulmonary venous capillaries or venules. Since high altitude pulmonary oedema is rapidly reversible with adequate oxygen therapy or removal to a lower altitude, one must postulate that any ob- structive lesion in the pulmonary venous capillaries must be capable of speedy resolution on relief from hypoxia. Fred and his associates (1962) concluded that the likeliest cause for the oedema was constriction of pulmonary veins induced by the hypoxia. This may be correct but does not take into account the possible effects of the intracapillary oedema vesicles which we have described in this paper and which we consider below.

Severinghaus (1971) believes there is no direct hypoxic damage to the lung in high altitude pulmonary oedema. $\mathrm{He}$ is of the opinion that the condition results from transarterial leakage giving rise to perivascular oedema. He refers to 'discrete focal damage' to pulmonary arteries allowing such transarterial leakage but does not describe the nature of this pulmonary vascular pathology. We are not familiar with a focal arterial lesion of this type in states of chronic hypoxia. Neither, apparently, is Arias-Stella (1971), who in discussing the hypothesis of Severinghaus notes that perivascular oedema and haemorrhage are not features of high altitude pulmonary oedema.

The oedema vesicles that we describe as projecting into the lumina of the pulmonary capillaries are in no way specific to high altitude pulmonary oedema. They represent localized collections of fluid in the fused basal membranes with detachment of the endothelial cells which are stretched over them. Such vesicles characterize the pulmonary oedema produced by inorganic or organic chemical compounds such as ammonium sulphate (Hayes and Shiga, 1970) and alpha naphthyl thiourea (Meyrick, Miller, and Reid, 1972). We have produced them in the pulmonary capillaries of rats fed on the seeds of Crotalaria spectabilis which contain the pyrrolizidine alkaloid monocrotaline (Kay, Smith, and Heath, 1969). Indeed, oedema vesicles in the thinnest portions of the alveolar wall over the convexities of the pulmonary capillaries have come to be regarded as characteristic of pulmonary oedema produced by toxic substances. Kay and Edwards (1973) studied the ultrastructure of the alveolarcapillary wall in four cases of mitral stenosis and found interstitial pulmonary oedema confined to the thicker portions of the alveolar wall containing collagen and reticulin fibres. It is clear from our present study that the hypoxia of simulated high altitude gives rise to oedema vesicles like those associated with toxic substances. The micropinocytotic vesicles that we have noted in the cytoplasm of the endothelial cells lining pulmonary capillaries and of the membranous pneumocytes 
are also found in mitral stenosis (Kay and Edwards, 1973).

One must be very cautious before ascribing functional significance to structural change in the absence of physiological data. However, we are of the opinion that since the lesions we have described are composed largely of oedema fluid they could form rapidly and thus account for the very rapid onset of the symptoms of high altitude pulmonary oedema (which one of us has experienced personally in the Peruvian Andes). Likewise, they could shrink equally rapidly on return to low altitude or on the administration of oxygen, both measures being known to produce speedy relief of symptoms. Furthermore, it is possible that these oedema vesicles could produce a haemodynamic effect by protruding into pulmonary venous capillaries. This would meet the requirements laid down by Fred et al. (1962) to explain the coexistence of acute pulmonary arterial hypertension and acute pulmonary oedema in the presence of normal left atrial and pulmonary venous pressure. One might object to the view that these oedema vesicles are of functional significance on the grounds that the pulmonary capillary bed has an enormous reserve capacity but we feel unable to assess the validity of this objection without knowing what percentage of capillaries are involved by these intraluminal projections. As morbid anatomists we can only point out that these microvascular lesions develop in rats with simulated high altitude pul- monary oedema and await investigation of their functional significance by physiologists.

Professor Heath acknowledges with gratitude the receipt of a research grant from the Nuffield Foundation.

\section{REFERENCES}

Arias-Stella, J. (1971). Discussion of paper by Severinghaus, J. W. quoted below.

Fred, H. L., Schmidt, A. M., Bates, T., and Hecht, H. H. (1962). Acute pulmonary edema of altitude. Clinical and physiologic observations. Circulation, 25, 929.

Hayes, J. A. and Shiga, A. (1970). Ultrastructural changes in pulmonary oedema produced experimentally with ammonium sulphate. Journal of Pathology, 100, 281.

Kay, J. M. and Edwards, F. R. (1973). Ultrastructure of the alveolar-capillary wall in mitral stenosis. Journal of Pathology. (In press).

- Smith, P., and Heath, D. (1969). Electron microscopy of Crotalaria pulmonary hypertension. Thorax, 24, 511.

Marticorena, E., Tapia, F. A., Dyer, J., Severino, J., Banchero, N., Gamboa, R., Krüger, H., and Peñaloza, D. (1964). Pulmonary edema by ascending to high altitudes. Diseases of the Chest, 45, 273.

Meyrick, B., Miller, J., and Reid, L. (1972). Pulmonary oedema induced by ANTU, or by high or low oxygen concentrations in rat-an electron microscopic study. British Journal of Experimental Pathology, 53, 347.

Severinghaus, J. W. (1971). Transarterial leakage: a possible mechanism of high altitude pulmonary oedema. High Altitude Physiology: Cardiac and Respiratory Aspects; A Ciba Foundation Symposium, p. 61. Churchill Livingstone, Edinburgh and London.

Toner, P. G. and Carr, K. E. (1971). Cell Structure, 2nd edition. p. 8. Churchill Livingstone, Edinburgh. 